\title{
Visual Analysis of Bioelectric Fields
}

\author{
Xavier Tricoche, Rob MacLeod, and Chris R. Johnson \\ Scientific Computing and Imaging Institute \\ University of Utah \\ tricoche@sci.utah.edu, macleod@cvrti.utah.edu, crj@sci.utah.edu \\ http://www.sci.utah.edu
}

\section{Introduction}

The bioelectric activity that takes place throughout the human body is essential to life. Electric, magnetic, and electromagnetic fields are produced by living cells that enable the action of muscles and the transmission of information in nerves. A variety of imaging modalities have therefore been developed to assess this activity. In particular, electrocardiography for the heart and electrocardiography and magnetoencephalography for the brain allow one to measure non-invasively the resulting electric signal. Beyond their clinical applications these techniques also provides the basis for a computational reconstruction of the physiological activity at the origin of this signal through the numerical solution of so-called inverse problems [9, 1].

To support the multiple bioengineering and biomedical applications that investigate the link between bioelectricity and physiology powerful postprocessing tools are necessary that facilitate the interpretation of measured and simulated bioelectric field data and permit one to derive anatomical and functional insight. In contrast to scalar quantities, which have been the traditional focus of biomedical visualization and for which effective depictions generally exist, the vector-valued bioelectric field information bears specific challenges that are insufficiently addressed by the visualization tools typically available to biomedical practitioners. In particular, depictions restricted to scalar attributes derived from bioelectric vector fields essentially deprive the analyst of the wealth of structural contents that is encoded by a field and its flow. Here, the notion of flow is defined mathematically and is used as a conceptual means to provide a continuous geometric interpretation to a smooth vector field, which lends itself to expressive representations. This type of electric or magnetic flow is obviously different from the fluid flows encountered in engineering applications of fluid dynamics, which have been the focus of most of the vector visualization methods developed within the scientific visualization community $[13,8]$. Yet, a common mathematical formalism offers an 
interesting opportunity to explore the applicability and potential extension of existing flow visualization techniques to bioelectric field problems.

In this context, the present paper describes the mathematical and algorithmic aspects involved in the application of advanced vector visualization techniques to the visual analysis of bioelectric fields. The focus is on electric fields and electric current densities associated with the cardiovascular and cerebral activity in humans. The data considered in the examples shown hereafter were acquired through the solution of so-called forward problems, in which 3D bioelectric fields are computed for a known bioelectric source [6]. The solution of these numerical problems is instrumental in the solution of inverse problems, through which researchers try to reconstruct the source activity at the origin of the signals measured non-invasively on the body surface.

The contents of the paper are organized as follows. Section 2 introduces some basic notions of bioelectricity and explains the relationship between the corresponding scalar, vector, and tensor quantities. Section 3 provides an overview of advanced flow visualization techniques. Section 4 demonstrates the potential of these methods for cardiovascular research while section 5 comments on corresponding results in the context of brain research. We conclude the presentation by summarizing the main results introduced in the paper in section 6 .

\section{Basic Notions of Bioelectricity}

For completeness, we introduce elementary notions of bioelectricity. The equations hereafter clarify the interconnections between scalar, vector, and tensor quantities that appear in the corresponding equations. Given the complexity of this topic, our ambition here is obviously limited and we restrict the presentation to an informal summary of basic notions that underlie the existence of bioelectric potentials and currents. For a more rigorous treatment of the subject we refer the reader to $[10,14]$.

\subsection{Physical laws}

The Maxwell's equations are fundamental relations that link the electric field $\mathbf{E}$, the electric flux density (or electric displacement) $\mathbf{D}$, the electric current density $\mathbf{J}$, the magnetic field $\mathbf{H}$, the magnetic flux density (or magnetic induction) $\mathbf{B}$, and the electric free charge density $\rho$ (bold letters denote vector quantities). Their differential form is as follows [14].

$$
\begin{aligned}
\nabla \times \mathbf{E} & =-\frac{\partial \mathbf{B}}{\partial t} \\
\nabla \times \mathbf{H} & =\mathbf{J}+\frac{\partial \mathbf{D}}{\partial t}
\end{aligned}
$$




$$
\begin{aligned}
& \nabla . \mathbf{D}=\rho \\
& \nabla . \mathbf{B}=0
\end{aligned}
$$

Additionally, current density and electric field are related through following equation.

$$
\mathbf{J}=\sigma \mathbf{E},
$$

where $\sigma$ corresponds to the electric conductivity, which is a second-order tensor field in general and reduces to a scalar field if the conductivity is homogeneously distributed. A number of simplifications can be made to these general formulae that take into account specific properties of the considered electromagnetic phenomenon. In particular, the bioelectric fields related to the cardiac and cerebral function are consistent with the assumption of timeindependence. The above equations reduce to following expressions for static of quasi-static problems.

$$
\begin{aligned}
\nabla \times \mathbf{E} & =\mathbf{0} \\
\nabla \times \mathbf{H} & =\mathbf{J} \\
\nabla . \mathbf{D} & =\rho \\
\nabla . \mathbf{B} & =0
\end{aligned}
$$

Hence, the electric field can be expressed as the gradient of an electric potential $\Phi$ :

$$
\mathbf{E}=-\nabla \Phi
$$

The relationship between the electric field and the resulting current flow of charged particles $\mathbf{J}$ is then defined by Ohm's law.

$$
\mathbf{J}=\sigma \mathbf{E}=-\sigma \nabla \Phi,
$$

If electric sources are present in the medium, they can be expressed in terms of a source density $I_{\nu}$. Since the divergence operator measures the net outflow per unit volume, this source density is linked to the current density by following equation.

$$
\nabla . \mathbf{J}=I_{\nu}
$$

Combining Equation (11) and Equation (12) we finally obtain the fundamental Poisson equation, which links scalar, vector, and tensor quantities in a single expression.

$$
\nabla . \mathbf{J}=I_{\nu}=-\nabla \cdot(\sigma \nabla \Phi)
$$

\subsection{Bioelectric potentials and impulse propagation}

Biological tissues behave as an electrolyte. In other words, they are conductive media in which ions play the role of charge carriers. Sodium, potassium, calcium, and chlorid are present in concentrations that vary significantly between 
the intracellular and extracellular media of excitable cells, most notably nerves and muscles. The corresponding concentration gradient generates a diffusion flow of ions across the cell membrane. Another flow exists that is induced by the action of an electric field on the potential created by the charges associated with the ions.

The thin cell membrane controls the exchange of ions between intracellular and extracellular domains. One building block of the membrane are proteins called pumps that consume energy to maintain the concentrations of sodium and potassium on both sides of the membrane, and that against the flow caused by concentration gradient. On the opposite, channels use energy to permit the flow of ions down the concentration gradient in a very controlled way. The resulting selective permeability is modified in response to the presence of an electric field and allows for fast changes of the transmembrane potential.

At rest, excitable cells have a negative potential, which is called polarized state. When a stimulus reaches the cell, the behavior of the channels is modified. The flow of positive charges into the cell is suddenly facilitated, leading to a rapid potential increase. This is called an action potential and corresponds to a depolarization of the cell. Once the potential reaches a threshold value the behavior of the channels changes and the inflow of positive charges is interrupted. The pumps are then used to transfer ions in and out of the cell, which eventually returns to its polarized state. For long thin fibers, the action potential affects only a limited patch of the membrane at a time. This impulse propagates by subsequently affecting the potential of adjacent patches. This results in their depolarization and regenerates another action potential cycle down the fiber. This propagation mechanism applies to nerves and individual muscle fibers, but also to more complex structures like nerve trunk and cardiac muscle.

\subsection{Data acquisition}

Non-invasive measurement techniques are essential in clinical practice. Electrocardiography (ECG) monitors cardiac activity by recording the evolution of the electric potential on the chest induced by successive excitation and repolarization waves occurring in heart. At a cellular level, depolarization and repolarization are linked to a phenomenon called action potential under which the varying permeability of the cell membrane entails a rapid increase of the cell potential (excitation or depolarization) that is followed by a decrease back to its original value (repolarization or recovery). The corresponding impulse propagates along muscular bundles, generating waves that are responsible for the electric signals measured on the body surface. ECG is used to detect cardiac arrhythmia (e.g. a skipped heart beat). It is also instrumental in the diagnosis of ischemia, a condition corresponding to a shortfall of the blood supply of the heart which reduces its pump function and can eventually lead to a heart attack. Similarly, the activity of the brain can be monitored through 
Electroencephalography (EEG), which records brain waves through the resulting variations of the electric potential on the scalp. In this case the electric signal is much weaker than the one produced by the heart and individual action potentials cannot be detected. Instead EEG measures the signal that arises form the synchronized bioelectric activity of a large number of neurons. This signal exhibits patterns of different frequencies that are related to different mental activities as well as pathologies. The latter includes epilepsy and Alzheimer's disease among others.

Computationally, a precise anatomical modeling combined with the use of realistic values for the conductivity of individual anatomical structures can be used to simulate bioelectric activity. Two types of computation exist. The forward problem consists in solving for the potential on the body surface when bioelectric sources are known. Conversely, inverse problems correspond to the localization of the source for which the boundary signal is known. Inverse problems are typically ill-posed and assumptions regarding the source model, the approximate location of the sources, and their number have to be made to obtain a unique solution. Source reconstruction by solution of an inverse problem is a prominent goal of the non-invasive techniques mentioned previously. Observe that the solution of the forward problem is instrumental in the solution of the corresponding inverse problem. The forward problem is typically solved numerically using either the Finite Element Method (FEM), the Finite Volume Method, or the Boundary Element Method.

\section{Visualization of Vector-Valued Data}

The depiction of the vector data that is ubiquitous in scientific problems has always been a major research focus topic for the scientific visualization community. The primary focus of the techniques created for that purpose has traditionally been on problems generally related to the engineering applications of fluid dynamics, e.g. automotive industry, aircraft design, and turbomachinery. In contrast, the vector-valued contents of biomedical datasets has received, comparatively, very little attention from visualization practitioners. As a consequence, a striking disconnect exists between the advanced vector visualization methods devised after several decades of dedicated research and the tools typically available to biomedical researchers. This section offers a brief introduction to three major categories of such advanced techniques, deferring their application to bioelectric fields to subsequent sections.

\subsection{Texture representations}

Texture-based techniques constitute a standard choice to generate intuitive visualizations of steady flow data. In contrast to depictions based on a set of discrete geometric primitives, the resulting dense representation is a powerful 
way to convey essential patterns of the vector field while avoiding the tedious task of seeding individual streamlines to capture all the structures of interest. The basic idea of most of the corresponding methods consists in applying a one-dimensional low-pass filter to an underlying white noise image. The filter kernel is here defined over individual streamline trajectories at each pixel of the image, thus creating patterns of strongly coherent colors that are aligned with the underlying continuous flow. At the same time, a strong contrast is preserved along directions orthogonal to the vector field orientation, which results in intuitive pictures that mimic a dense coverage of the domain with thick streamlines. A wide variety of techniques have been proposed following this basic principle [8]. Originally applied to planar flows, these algorithms were later extended to vector fields defined over curved surfaces [7, 18].

\subsection{Topological methods}

Topological methods apply to the visualization of vector fields a mathematical framework developed for the qualitative study of dynamical systems [11]. This approach was introduced in the fluid dynamics community in an effort to provide a rational language to describe complex three-dimensional flow patterns observed in experimental flow visualizations. So-called critical points (where the magnitude of a vector field vanishes) are the nodes of a graph that segments the domain into regions of qualitatively different behaviors. The boundaries between neighboring regions called separatrices are streamlines or stream surfaces.

This technique was introduced in flow visualization $[4,3]$ in the form of methods that construct the topological graph for two-dimensional flows and laid the basis of an extension to three-dimensional flows. This seminal work stimulated numerous subsequent publications [15]. Of particular interest for the applications considered in the following is the extraction of the topological graph of a vector field defined over a curved boundary. The challenging aspect of this setting lies in the fact that the corresponding geometry is not smooth and the resulting discontinuities of the tangent plane lead to some specific topological features that must be accounted for in the visualization. In particular, the integration of separatrices can be done using the technique proposed by Polthier and Schmies [12], which is built upon the notion of geodesic on polygonal surfaces.

\subsection{Stream surfaces}

Stream surfaces are formed by the advection of a (seed) curve along the flow. Hence, they embed in a continuous depiction the information conveyed by an infinite number of streamlines and enhance the understanding of depth and spatial relationship. They can be computed either implicitly [17] as isosurfaces of a pre-computed scalar function, or explicitly through numerical integration [5], which offers more flexibility in the choice of the initial curve 
and yields a geometric representation of better quality. The basic principle of this latter approach consists in propagating a polygonal front by integrating the trajectories followed by its discrete vertices. A divergent behavior of neighboring vertices can be dynamically accounted for by adaptively inserting additional vertices. The triangulation of the surface spanned by this motion is constructed on the fly. We recently introduced a method that allows a more precise control over the tessellation of the surface and creates stream surfaces of high visual quality, even in the challenging case of turbulent flows [16, 2].

\section{Visualization of Cardiac Bioelectricity}

The dataset that we consider in this section consists of a forward Finite Element computation of the electric potential $\Phi$ over the volume of a torso. The general context of this computation is a research project aimed at gaining new insight into the mechanisms and consequences of myocardial ischemia through the analysis of experiments and simulations. Myocardial ischemia corresponds to a shortfall in blood supply, which arises when blood supply to the heart does not meet demand, leading to chest pain (angina) and reduced pump function. In the extreme, a complete blockage of blood leads to a heart attack. The FEM method is chosen for the flexibility it provides in the choice of the torso geometry (the heart surface - the epicardium - constitutes here an internal boundary) and the distribution of tissue conductivity. The source information for the forward problem on the epicardium stems from experimental measurements carried out by Dr. Bruno Taccardi at the Cardiovascular Research and Training Institute (CVRTI) at the University of Utah. The elements used are linear tetrahedra. The resulting current density (obtained by derivation of the electric potential) is piecewise constant over the mesh and an averaging over the control volume (i.e. the direct neighborhod) of each node combined with piecewise linear interpolation yields the projected value of the current density field in a $C^{0}$ space suitable for flow integration methods.

\subsection{Electric field over boundary surfaces}

The visualization techniques applied to the epicardium in cardiovascular research are typically restricted to representations of the electric potential, a scalar quantity that can be depicted using a combination of color coding and isocontours on the geometry of the heart. This picture can be enhanced by adding glyphs that show both the direction and the magnitude of the threedimensional electric field crossing the epicardium as shown in Fig. 1.

Additional information can be conveyed by the use of a dense, texturebased representation of the electric field defined over the surface of the epicardium. Visualizing the associated topological graph permits to indicate the

electric connectivity of the epicardium and highlight coherent structures of the current, which naturally complements the texture-based visualization. An 


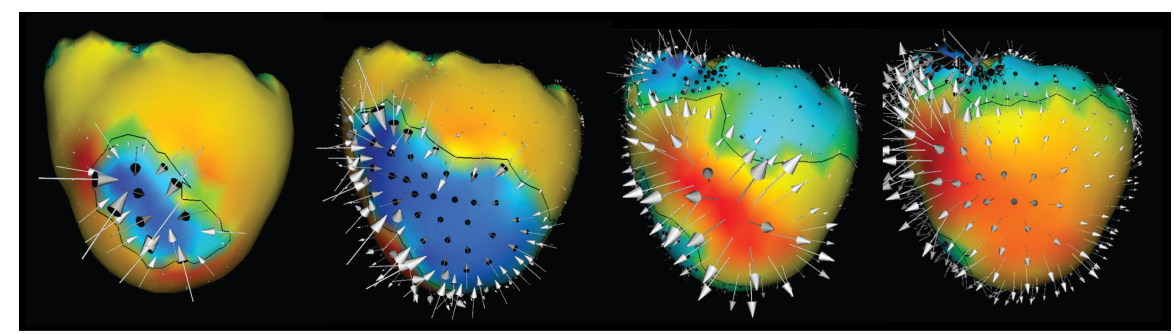

Fig. 1. Electric potential (color coding) and electric field (glyphs) on the epicardium. Left and middle left: excitation phase. Middle right and right: repolarization phase.

interesting question in this context corresponds to the potential interpretation of the topological features exhibited by the electric field in terms of what is known in cardiac electrophysiology as epicardial breakthrough. This phenomenon occurs when an activation wave in the cardiac tissue breaks through the surface, generating a local potential minimum on the epicardial surface, as shown in Fig. 2.
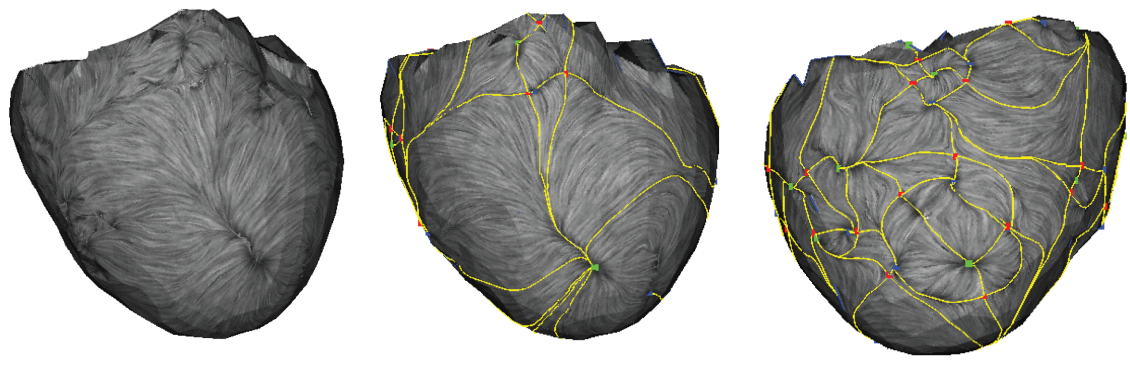

Fig. 2. Topology of bioelectric field on epicardium. The images show a texture representation of the potential gradient on the surface enhanced by the depiction of the associated topological graph. Green points correspond to potential minima, blue points mark potentials maxima. Left and middle images show an anterior view of the epicardium. Right image is seen from the left of the torso.

Similar pictures can be achieved for the torso surface. In that case the topological structure provides little additional information in the absence of clearly defined separatrices, which is due to the overall dipolar nature of the return current. However, significant lines can be extracted, along which the surrounding flow tends to converge as it moves toward the chest boundary or away from it. Texture representations can also be combined with a color coding of the electric potential to the show the interrelation between quantitative and structural aspects. They can also be computed over cutting planes as a means 
to unveil the patterns of the bioelectric return current in the chest volume. The use of transparency permits to mitigate the occlusion issues inherently associated with this type of depiction. It can be seen how the texture, through careful orientation of the corresponding plane, is able to capture the geometric signature of intricate three-dimensional structures, as shown in Fig. 3.
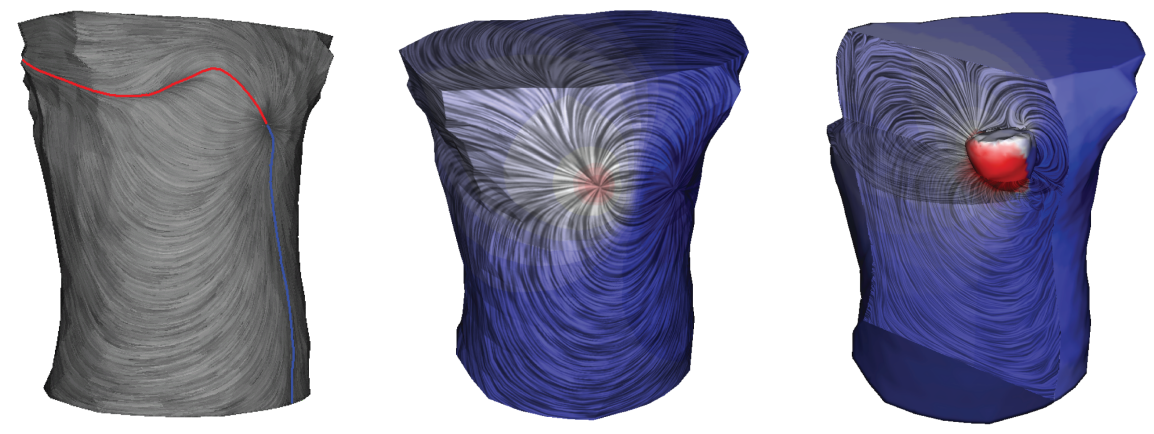

Fig. 3. Texture representations of the return current in the torso. Left: Visualization of the torso surface combined with lines along which the volumetric current attaches or detaches from the outer boundary. Middle: Texture combined with color coding of electric potential (negative values mapped to blue, positive values to red). Right: Two textures computed over cutting planes combined by transparency. The geometry of the heart remains opaque for context.

\subsection{Electric current in the torso}

Previous visualizations are limited to planes and curved surfaces. To offer further insight into the structural properties of the current inside the torso volume, stream surfaces can be used to probe the three-dimensional geometry of the current and inspect the resulting connectivity between distant locations distributed over the epicardium. The primary difficulty encountered when applying stream surfaces to explore 3D flow volumes lies in the choice of an appropriate seed curve. This curve must be chosen to capture interesting patterns. Because of the non-local nature of stream surfaces (their overall shape is determined by the numerical integration of the underlying vector field) finding a "good" seed curve is typically a difficult task that requires repeated tries to achieve satisfactory results.

To address this problem we leverage the dipolar nature of the cardiac source. The dipolar shape is namely a characteristic pattern of bioelectricity and surfaces can be used to visualize the resulting arches spanned by the return current across the volume. Specifically, we are interested in the interconnections between regions of inflow and outflow distributed over the epicardium. A way to investigate these connections consists in computing the 
dot product of the electric current with the surface normal on the epicardium and to select isocontours of the resulting flux (where positive values correspond to a source behavior while negative values indicate a sink) as seeding curves. The results are presented in Fig. 4. The arch-type connections induced by multiple dipoles as well as their intertwinement are clearly visible.
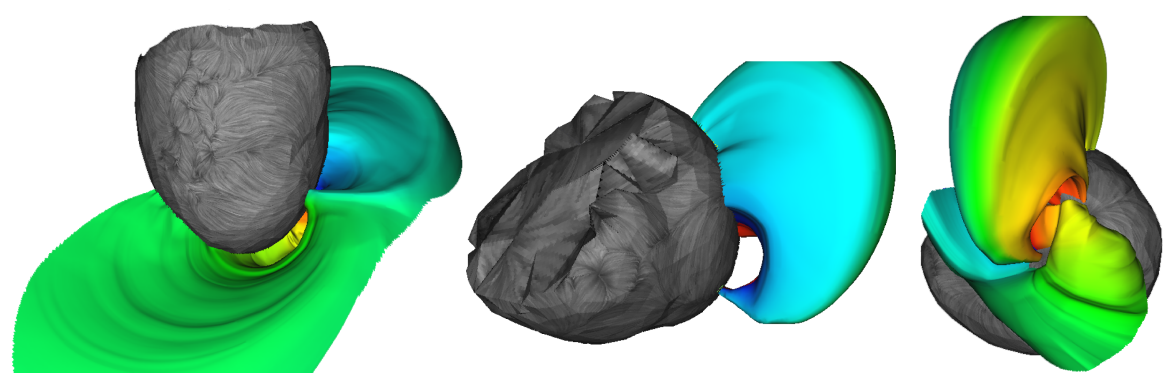

Fig. 4. Visualization of bioelectric field in the direct vicinity of epicardium with stream surfaces. The surfaces capture the geometry of the current induced by the dipole equivalent cardiac source. They also provide an effective representation of the interconnections that exist between different regions on the heart surface. The seeding curves correspond to isocontours of the electric flux selected close to local extrema. A rainbow color map is used along each seeding curve to visualize the stretching of the return current as it propagates through the torso.

An interesting extension of that type of representation would be to extract the full three-dimensional (and transient) electric connectivity of the epicardium.

\section{Visualization of Cerebral Bioelectricity}

The datasets considered in this section are all related to brain research and more specifically to the challenging problem of EEG source localization. The goal of the corresponding investigation is to assess the incidence of different source localizations and different models of white matter conductivity anisotropy on the solution of the forward problem. In particular, this modeling strategy impacts the accuracy of the solution to the inverse problem required for source reconstruction since forward problems are building blocks of the corresponding computation. Practically, the forward problem is solved over a high-resolution FEM mesh composed of tetrahedral elements. The modeling of tissue conductivity is based on a segmentation that comprises skin, skull, cerebrospinal fluid (CSF), gray matter, and white matter [19]. A number of computations were carried out for different positions of a dipole source, yielding the values of the resulting electric potential on the head surface, which corresponds to the signal that can be acquired non-invasively using EEG. 
Moreover, the white matter conductivity was modeled as anisotropic in some simulations and compared to the solution of the forward problem obtained under the assumption of an isotropic conductivity of the white matter.

The task of the visualization in that context is to help elucidate the intricate structure of the three-dimensional return current in the brain and more specifically the impact of source localization and conductivity modeling on the corresponding patterns. It is important to emphasize that the non-uniformity of the conductivity and its anisotropy (in other words its tensor nature) result in a non-trivial relationship between electric potential - which is the focus of most visualizations in this type of application - and return current. The contribution of vector visualization techniques is therefore to shed some light on this relationship. Practically, as in the case of the torso dataset considered previously, a piecewise linear interpolation of the electric potential over the mesh yields piecewise constant electric field and return current (the conductivity tensor is cell-wise constant as well), which are transformed in continuous vector fields by averaging the values over the control volume of each vertex and applying piecewise linear interpolation to the resulting point-wise quantities.

\subsection{Visualization of bioelectricity on the head surface}

As in the study of the heart, texture representations offer a natural means to visualize the return current on the boundary of the dataset, that is the head surface. Again, the natural complementarity between the directional information conveyed by the texture and the quantitative information (e.g. electric potential or current magnitude) encoded by a color map permits to offer a comprehensive picture of the bioelectricity on manifolds. Observe that the homogeneous Neumann boundary condition imposed on the simulation (i.e. the electric flux is zero across the head surface) justifies an analysis of the current restricted to boundaries since the vector field is tangent to the surface. The images shown in Fig. 5 reveal that the geometry of the return current is not affected by the conductivity model while its magnitude strongly varies between an isotropic and an anisotropic model.

\subsection{Visualization of bioelectricity in the brain}

As seen previously, texture representations can be used on cutting planes to help visualize the three-dimensional return current defined inside the brain. When applied to a dataset computed with an anisotropic model of white matter conductivity, this technique shows the asymmetry of the electric patterns, in strong contrast with the smooth and symmetric images obtained for isotropic models, as seen in Fig. 6. Notice that the canonical orientation of the planes corresponds to what is typically used in a clinical setting to visually assess the spatial distribution of scalar quantities. Moreover the planes are positioned so as to both contain the source position. 

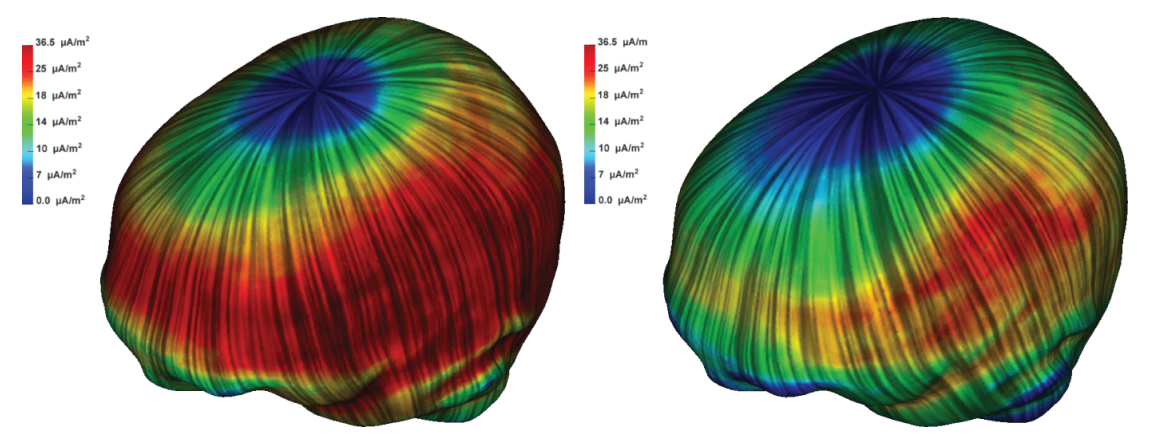

Fig. 5. Patterns of the surface return current corresponding to a source localized in the left thalamus. Left: an isotropic model has been used for the white matter. Right: a 1:10 anisotropic model has been applied to the white matter. The ratio corresponds to major vs. minor diffusion direction as inferred from a DTI scan. The color coding corresponds to the magnitude of the return current
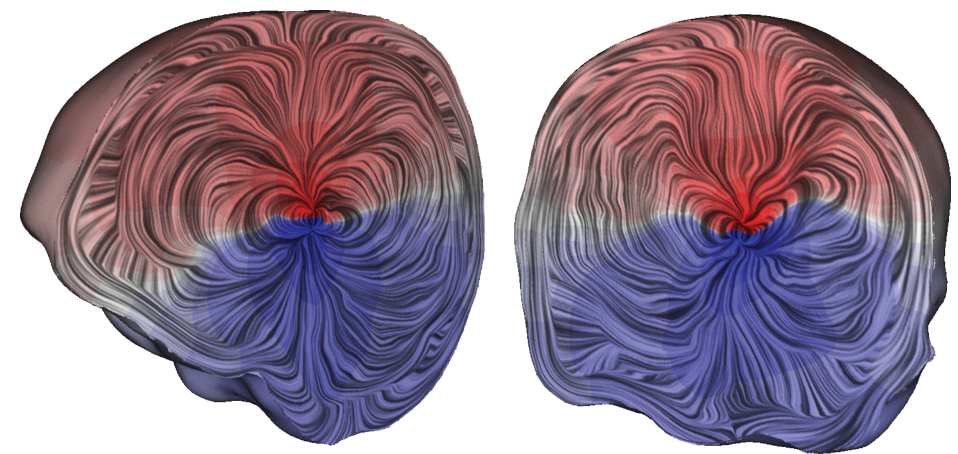

Fig. 6. Textures computed on sagittal and coronal clipping planes reveals details of the dipolar source and its interaction with the surrounding anisotropic tissue. The electric current is clearly diverted by the presence of white matter tracts that lie close to the source. The field also changes direction very rapidly as it approaches the skull just beneath the surface of the head.

The complex patterns exhibited by these textures suggest that the return current has a very convoluted structure that cannot be fully captured by planar representations. Stream surfaces can therefore be employed to gain deeper insight into this three-dimensional structure. Yet, the intricacy of the return current makes the seeding task even more challenging than it was previously in the case of the heart dataset. Moreover, the natural seeding strategy provided by the epicardium and isocontours defined over it has no straightforward equivalent in this context. A possible solution consists in introducing an artificial surface, e.g. a sphere, around the known position of the source and to apply the flux-based seeding strategy mentioned in the previous section to this sphere. Alternatively, isopotential lines can be computed on the head 
surface and isolines located in the direct vicinity of potential extrema can play the role of seed curves. We remark that the homogeneous Neumann boundary conditions would impose that any field line started on the head surface remains embedded on that surface. Yet, an arbitrarily small offset from the head surface guarantees that the return current leaves the surface in one direction. These different strategies are illustrated in Fig. 7.
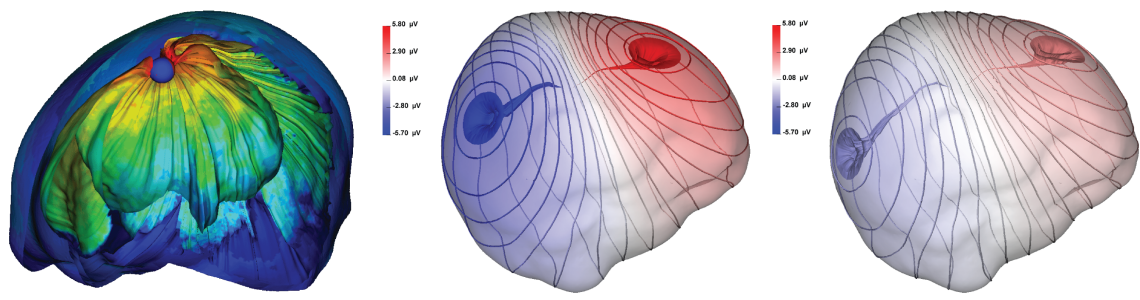

Fig. 7. Stream surface visualization of return current in the brain induced by dipolar source. Left: Seed curve corresponds to isocontour of electric flux on sphere enclosing the source (source located in left thalamus). Middle: Seed curves chosen as isocontours of the electric potential on the head surface (isotropic conductivity model, source in sematosensory cortex). Right: Same with 1:10 anisotropic conductivity model.

Because of the structural complexity of the return current and the lack of clearly defined symmetries (especially in the anisotropic case), visualizations composed of a few stream surfaces offer an intrinsically partial picture. Increasing the number of stream surfaces in an effort to achieve a dense filling of the space is faced with strong occlusion and visual clutter. A different solution therefore consists in using parameterized seed curves. In other words, the seed curve becomes a dynamic curve and as the underlying parameter changes continuously, the resulting transformation of the associated stream surface can be visualized as an animation. To apply this general principle to the brain dataset, a circle of increasing radius can be used as a parametric curve, centered around the dipolar source and lying in the horizontal plane orthogonal to the dipole axis. An analysis of the differences between isotropic and anisotropic conductivity of the white matter can then be made in a side by side comparison of the corresponding animations. This method is illustrated in Fig. 8.

\section{Conclusion}

Electric and magnetic fields are vector fields that represent bioelectric activity in the human body. Yet, their interpretation has been hampered by the challenges of visualizing them in meaningful ways. One result has been 


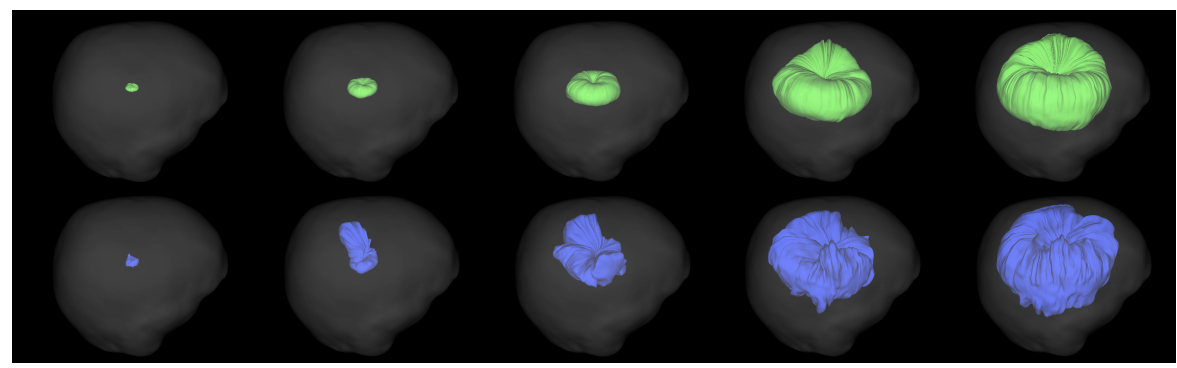

Fig. 8. Evolution of a stream surface integrated along the return current under the parameterization induced by the increasing radius of its seeding circle. Top row: Frames from an animation corresponding to isotropic white matter model. Bottom row: Frames of the animation obtained for anisotropic white matter model.

that visualization and analysis have focused on the scalar potentials (electric and magnetic), for which mature visualization strategies exist. In this paper we have presented a variety of results from our ongoing research, which concentrates on the deployment and targeted extension of vector visualization methods that offer a global and expressive depiction of the continuous flow fields underlying the discrete information in numerical data sets. Our preliminary results show that visualizations combining a representation of the bioelectric current with related scalar attributes like potential and current amplitude permit a deeper understanding of the three-dimensional shape of the bioelectric sources and their fields. They also offer new insight into the impact of tissue characteristics, e.g., anisotropy, on the resulting bioelectric fields.

\section{Acknowledgments}

The authors are greatly indebted to Drs. Bruno Taccardi and Frank Sachse from CVRTI at the University of Utah, as well as to Dr. Carsten Wolters from the University of Muenster, Germany, who have provided their close collaboration, fruitful discussions, and the data sets that were used in the examples studied in the paper. This work was supported by the Center for Integrative Biomedical Computing, NIH NCRR Project 2-P41-RR12553-07.

\section{References}

1. A.M. Dale and M.I. Sereno. Improved localization of cortical activity by combining EEG and MEG with MRI cortical surface reconstruction. J. Cognit. Neurosci., 1999.

2. C. Garth, X. Tricoche, T. Salzbrunn, T. Bobach, and G. Scheuermann. Surface techniques for vortex visualization. In Data Visualization 2004 (Proceedings of 
Eurographics/IEEE TCVG Symposium on Visualization 2004), pages 155-164, 2004.

3. A. Globus, C. Levit, and T. Lasinski. A tool for visualizing the topology if three-dimensional vector fields. In IEEE Visualization Proceedings, pages 33 40, October 1991.

4. J.L. Helman and L. Hesselink. Representation and display of vector field topology in fluid flow data sets. Computer, 22(8):27-36, 1989.

5. J.P.M. Hultquist. Constructing stream surfaces in steady $3 \mathrm{D}$ vector fields. In Proceedings of IEEE Visualization 1992, pages 171-178, October 1992.

6. C.R. Johnson. Numerical Methods for Bioelectric Field Problems, pages 162180. CRC Press, 1995.

7. B. Laramee, J.J. van Wijk, B. Jobard, and H. Hauser. ISA and IBFVS: Image space based visualization of flow on surfaces. IEEE Transactions on Visualization and Computer Graphics, 10(6):637-648, nov 2004.

8. R.S. Laramee, H. Hauser, H. Doleisch, B. Vrolijk, F.H. Post, and D. Weiskopf. The state of the art in visualization: Dense and texture-based techniques. Computer Graphics Forum, 23(2):143-161, 2004.

9. R.S. MacLeod and D.H. Brooks. Recent progress in inverse problems in electrocardiology. IEEE Engineering in Medicine and Biology Magazine, 17(1):73-83, Jan/Feb 1998.

10. R. Plonsey and R. C. Barr. Bioelectricity, A Quantitative Approach. Second Edition. Kluwer Academic / Plenum Publisher, 2000.

11. H. Poincaré. Sur les courbes définies par une équation différentielle. J. Math. 1:167-244, 1875. J. Math. 2:151-217, 1876. J. Math. 7:375-422, 1881. J. Math. 8:251-296, 1882.

12. K. Polthier and M. Schmies. Straightest geodesics on polyhedral surfaces. pages 391-408, 1998.

13. F.H. Post, B. Vrolijk, H. Hauser, R.S. Laramee, and H. Doleisch. The state of the art in flow visualization: Feature extraction and tracking. Computer Graphics Forum, 22(4):775-792, 2003.

14. F.B. Sachse. Computational Cardiology: Modeling of Anatomy, Electrophysiology, and Mechanics. Springer-Verlag New York, May 2004.

15. G. Scheuermann and X. Tricoche. Topological methods in flow visualization. In C.R. Johnson and C. Hansen, editors, Visualization Handbook, pages 341-356. Academic Press, 2004.

16. X. Tricoche, C. Garth, T. Bobach, G. Scheuermann, and M. Ruetten. Accurate and efficient visualization of flow structures in a delta wing simulation. In Proceedings of 34 th AIAA Fluid Dynamics Conference and Exhibit, AIAA Paper 2004-2153, 2004.

17. J.J. van Wijk. Implicit stream surfaces. In Proceedings of IEEE Visualization '93 Conference, pages 245-252, 1993.

18. D. Weiskopf and T. Ertl. A hybrid physical/device space approach for spatiotemporally coherent interactive texture advection on curved surfaces. In Proceedings of Graphics Interface 2004, pages 263-270, 2004.

19. C.H. Wolters, A. Anwander, X. Tricoche, D. Weinstein, and R.S. MacLeod. Influence of tissue conductivity anisotropy on EEG/MEG field and return current computation in a realistic head model: A simulation and visualization study using high-resolution finite element modeling. NeuroImage, 30(3):813-826, 2006. 